\title{
EcoClass - An easy-to-access GUI for LCA data to assess product utilization schemes
}

\author{
Volker Barth ${ }^{*}$, Jens Krefeldt ${ }^{1}$, Jan-Christian Marinesse ${ }^{2}$ \\ GELENA, Carl von Ossietzky University, Faculty 2, D-26111 Oldenburg \\ *Corresponding author: volker.barth@uni-oldenburg.de \\ ${ }^{1}$ now at: TOJAQ GmbH, Breite Str. 39, D-38159 Vechelde \\ ${ }^{2}$ now at: OpenKnowledge GmbH, Bismarckstr. 13, D-26122 Oldenburg
}

\begin{abstract}
We present experiences from using the EcoClass life cycle assessment tool in participatory product development. EcoClass is designed as an easily accessible, user-friendly tool providing data on three emissions categories during production and utilization of urban transport vehicles. In contrast to most other LCA models, EcoClass allows online modifications of vehicle utilization parameters to reflect changes in behavior. Its use with lay people revealed that the model in its current version is well suited to raise awareness and provide information, whereas it still needs improvement before it can be used for detailed production decisions.
\end{abstract}

\section{Introduction}

In recent decades, life-cycle assessment (LCA) has become a frequently used method in product development to assess ecological impacts of products or production methods. Addressing the ecological impacts of all processing steps from resource extraction to disposal/recycling requires large databases of technologies, inputs and by-products, and their associated emissions. Currently available software products to work on these databases are highly specialized tools that require expert knowledge and training in order to produce meaningful results.

Insights from innovation research indicate, however, that inclusion of non-experts like consumers during product development may be advantageous: companies take customers' needs and environmental concerns more seriously [van den Ven 1986], and combining the technical knowledge of companies with the contextual every-day knowledge of customers may lead to products that fit better to customers' needs [Lester et al. 1998]. However, consumer participation is less ambitious than political participa- 
tion and aims mainly at increasing consumer satisfaction, commitment to the company, and market success [Hansen and Raabe 1991].

With rising environmental concerns among average consumers, the necessity to address environmental impacts already during the design phase becomes an important issue. LCA models offer the data and the methodology to assess these impacts in a comprehensive manner. However, the integration of LCA models in participatory product development processes is still challenging for mainly two reasons: consumers usually do neither have the expert knowledge to run usual LCA models nor are they used to use computer models at all, while existing models are neither designed nor being flexible enough to be used by non-experts in participatory processes. (This of course applies not only to LCA models; see e.g. Kasemir et al. [2003] for experiences with climate change models.)

We present the EcoClass model that has been used by lay users for participatory development of climate-friendly products according to the INNOCOPE guidelines in a pilot study in 2005 [Hoffmann 2006]. These guidelines feature model support to address and assess the ecological impacts of the product to be developed. The paper describes the main model features and reflects on its use during the INNOCOPE process.

\section{The INNOCOPE product development process}

The INNOCOPE process (INNOvation through COnsumer-integrated Product dEvelopment, [Hoffmann 2006]) consists of a series of three workshops within approximately six months. Participants are consumers as well as company representatives from different divisions. When recruiting consumers care is taken to ensure diversity of social and demographic background and of affinity towards the product to develop, which was determined by the company in advance. During the first workshop the participants generate product ideas, which are evaluated, substantiated and refined in two subsequent meetings by using professional creativity and moderation techniques. The company is expected to take up ideas from the participants and work on their realization in between the workshops to provide feedback on workshop results from the production side, and to allow for reflection and discussion among the participating consumers.

The INNOCOPE method was first employed in a pilot study in 2005, where some 15 consumers and 4 company representatives developed a pedelec ('pedal electric cycle', a bicycle where pedaling is supported by an electric motor). In the first workshop, awareness for environmental issues was raised by an expert talk focusing on climate change and the environ- 
mental impacts of traffic. In the second workshop, participants used the EcoClass model (see section 3) to evaluate the environmental impacts of their current traffic behavior, and to compare the impacts of different means of transportations in more detail. It was also possible to evaluate the effects of substituting one or more vehicles in a mobility chain.

\section{The EcoClass model}

The EcoClass model (ECOlogical and CLimatic ASsessment Software, available at http://www.gelena.net) in its current version 0.2 is a tool to assess the environmental impact of vehicles used in urban personnel transport. Its purpose to visualize alternative ways to build and operate the product developed in the INNOCOPE process.

\subsection{Database}

To allow for greater flexibility to apply the final software we use a broad database covering lots of products and processing steps, which can easily be extended with few additions once a particular product is selected. We use the GEMIS database included in the LCA model GEMIS [Fritsche et al. 1989, 1994] in version 4.2 (http://www.oeko.de/service/gemis/).

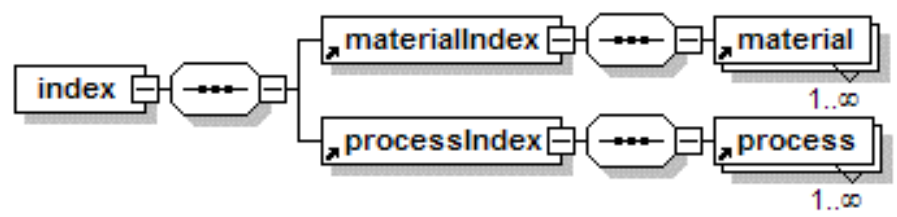

Fig. 1. An index XML file is loaded at application startup and acts as a memorysaving proxy to materials and processes. The material and process proxy elements have XML attributes (not shown here for complexity reasons) with values "pointing” to XML files containing the actual data. Their structure is shown in Figure 2.

EcoClass is written in Java to ensure availability on most major computer platforms. The proprietary database format of the Windows-based GEMIS model was converted to XML by exporting the database to the Microsoft Access format and extracting this using Java's ODBC interface. During this process care was taken to preserve the structure of the GEMIS database, although few ambiguities had to be resolved manually, and extraction of cost information has been skipped. Figures 1 and 2 show the structure of the XML database elements forming a meta model. 


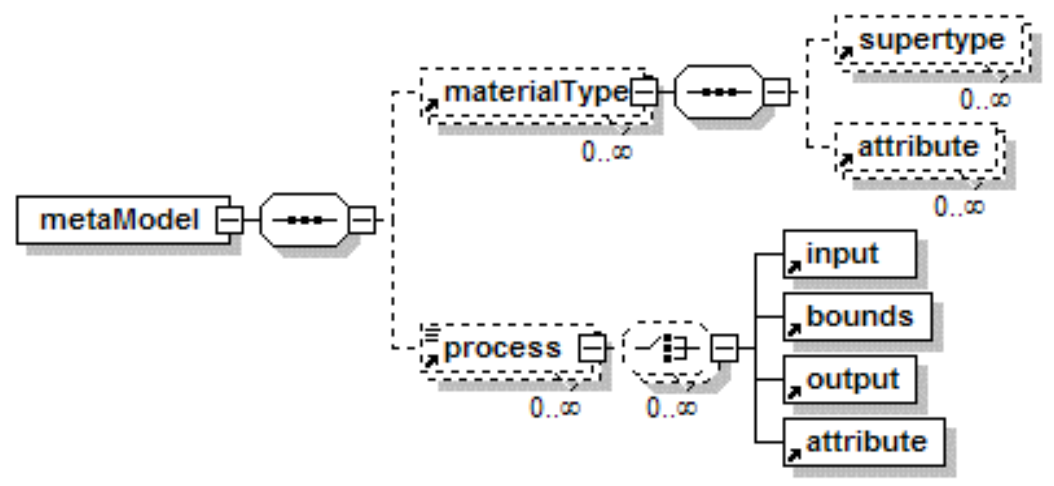

Fig. 2. The materialType and process elements including their subelements represent the meta model for EcoClass' product system (XML attributes not shown here for complexity reasons).

\subsection{User Interface}

The graphical user interface (GUI, see Figure 3) of EcoClass is a plugin for the Eclipse framework (www.eclipse.org). Main area of interaction is a graphical editor that employs the Graphical Editing Framework of Eclipse. Within this editor, users construct so-called product systems by selecting predefined production or utilization processes from the database and connecting the outputs of one process with the inputs of other processes in order to build a production chain. Inputs and outputs are materials or assembled products. The central process is a utilization process combining an input (e.g. car) with the energy input to operate it (e.g. gasoline) to produce one service unit as output, e.g. one person-kilometer (pkm). The amount of service units to be delivered in the given setup is defined separately.

Utilization processes extend standard production processes (from the GEMIS database) to provide a user interface to specify the operation mode of the product in a parameterized manner. In EcoClass 0.2, vehicles may differ in the passenger count, the road mix, or the driver's style of driving. Manipulating these specifications affects the energy use (and thus emissions) per service unit, the actual relation being either derived exactly (passenger count), or based on simple extrapolations (driving style, road mix). This feature allows the user to directly monitor different usage conditions (although at reduced accuracy), whereas in most other LCA models this can only be achieved by setting up a completely new process offline. Of course, this latter method can also still be employed in EcoClass.

Utilization processes in EcoClass have pre-selected input processes, so that it is usually only necessary to select a utilization process and specify 
the amount of service units that shall be delivered. It is, however, possible to manually exchange inputs of electric energy, e.g. to compare emissions of a pedelec whose batteries are charged with average household electricity with one that is running on green electricity. It is also possible to display all input processes of a given process, and the input processes of the input processes and so on, so as to retrace the whole production chain as defined in the GEMIS database.

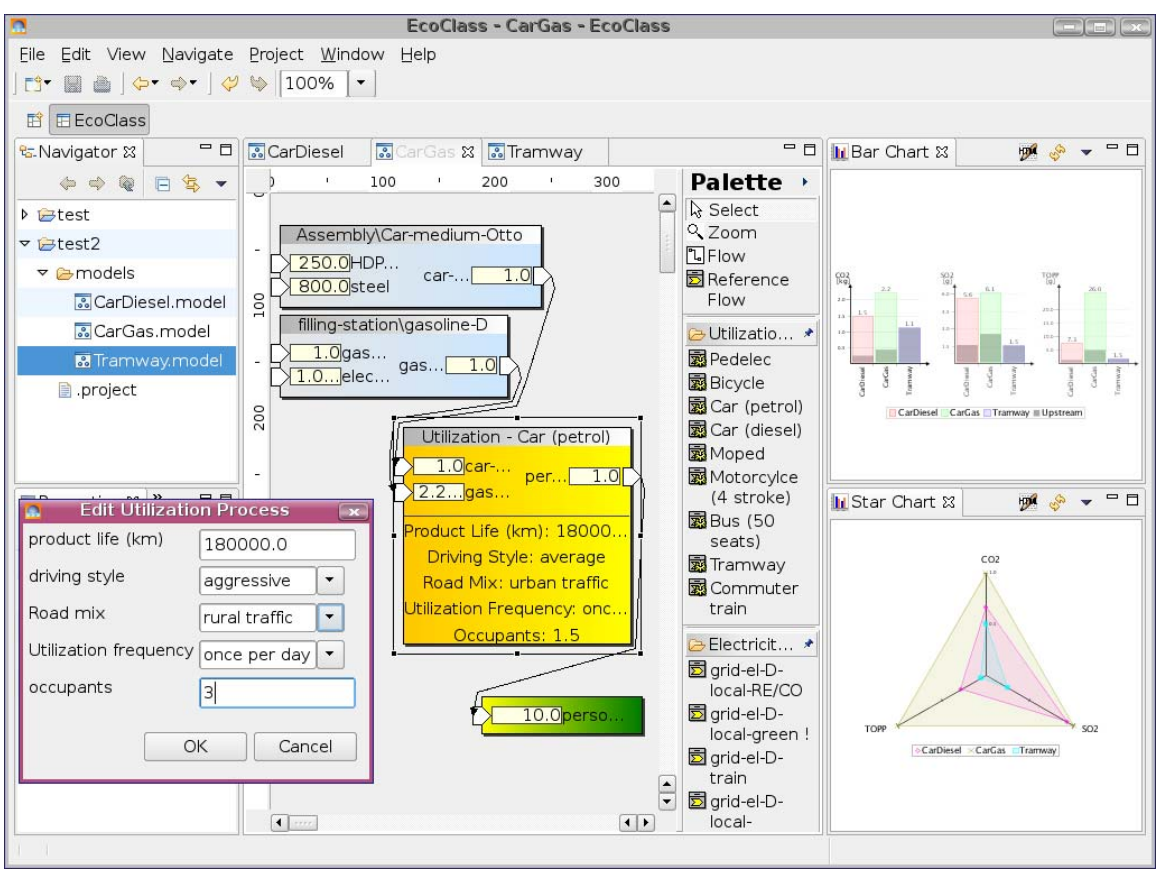

Fig. 3. Screen shot of the EcoClass GUI. The central part shows the graphical editor containing a product chain with one utilization process. The two smaller boxes above represent the processes that provide the inputs of the utilization process. The little box right below the utilization process is used to define the amount of service units to be used. Some of the utilization parameters have just been changed in the dialog box in the lower left of the screen shot and will be transferred to the utilization process after clicking "Ok”. Graphical visualization of results is shown on the right. The user may rearrange all parts of the GUI.

\subsection{Calculation of Results}

The GEMIS database provides two sets of emissions associated to a given process: those actually emitted during execution of that process (direct emissions), and the total emissions that have been set free over the whole 
process chain. Using this second set allows to calculate the emissions "from the end" of a process chain and to skip the tedious explicit setting up of all steps that are required to build the final product. This is helpful when only the utilization phase shall be examined since the user can concentrate on product operation. On the other hand, this restricts the model to the process chains (and the emissions) that are predefined in the database; it is not possible to assess production alternatives during the construction of the product. Therefore, the model loses much of the characteristics of a fully featured LCA tool. Nevertheless, this approach was chosen for the first versions of EcoClass, which should focus on product utilization. Full LCA functionality is scheduled to be available in a future EcoClass version 1.0.

To address the environmental impact of products, EcoClass calculates emissions of greenhouse gases, of gases that cause acid rain, and of precursor gases of tropospheric ozone, the major component of photochemical smog. These quantities are expressed as the equivalent amount of carbon dioxide that would cause the same additional greenhouse effect $\left(\mathrm{CO}_{2}\right.$ equivalent), the equivalent amount of sulfur dioxide that would lead to the same acidification ( $\mathrm{SO}_{2}$ equivalent), and the amount of ozone that will result from the ozone precursor gases (TOPP equivalent), respectively. Other environmental impacts like resource use, waste, or water pollution are neglected to reduce complexity, as these are less important for vehicles.

The actual calculation proceeds in the same manner for each of the three emission categories $\mathrm{CO}_{2}$ equivalents, $\mathrm{SO}_{2}$ equivalents, and TOPP equivalents. The emissions in a given category associated to one person, which drives a specified distance in a particular vehicle is derived by adding the direct emissions from vehicle utilization over the specified distance to the proportional share of total emissions from producing the vehicle and from producing the energy (e.g. fuel) to operate it.

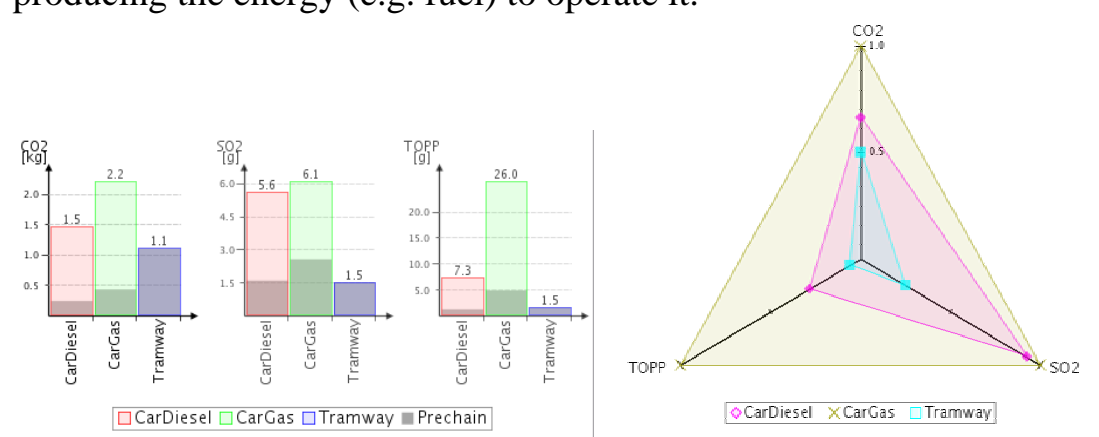

Fig. 4. Bar chart (left) and spider diagram for three sample product systems, representing a diesel-fueled car, a gasoline-fueled car, and a tramway. Shaded boxes within the bars of the bar chart indicate emission from the production of vehicle and fuel/energy. 


\subsection{Visualization of Results}

The calculated results are displayed in two graphical representations (see Figure 4): a bar chart to compare absolute emission values, and a spider diagram to compare relative emissions between product systems. In the bar chart, emissions are grouped by emissions categories, so that differences between product systems are displayed in absolute terms. Additionally, the amount of "gray emissions", which arise from the production of vehicle and fuel/energy, respectively, is shown in order to visualize the share of emissions that can be influenced by users' behavior. In contrast to that, the spider diagram shows three axes representing the three emissions categories. The maximum emission value in a given category is assigned the value 1 , while the emissions from other product systems are displayed relative to that. This allows for easy comparison of product systems.

\subsection{Comparison with other LCA tools}

A number of other LCA tools also claim to be user-friendly, for example SimaPro (www.pre.nl/simapro/) or $\mathrm{GaBi}$ (www.gabi-software.de). A closer look reveals some limitations of their usefulness for interactive use with lay participants. Often, user have to leave the graphical process chain editor to input data via large spreadsheets. Simple parameterizations of behavior are not available - instead, quantities like energy use have to be entered (and known) in absolute values. As this enhances precision it is preferable when users have some minimum specialized knowledge of the product, like staff of the producing company. Despite of the reduced precision that makes EcoClass hardly suitable for applications like expert opinions, it gives access to LCA technologies to lay users with only common knowledge, which is hardly sufficient for usual LCA tools.

\section{Workshop Experiences}

EcoClass was used by lay people during the second workshop in the INNOCOPE process. After listening to a plenary presentation and introduction to the software, participants split into three smaller working groups of about 5 persons to use the model by themselves. Each group was equipped with one PC with two monitors, so that the actions of each user could be seen and possibly commented on by the others. The default task was to reflect on personal traffic behavior by assessing the emissions of day-to-day trips and to compare them to trips where a pedelec is used on 
suitable sections. Users were, however, free to use the software for other investigations as well. If required, users could get assistance in operating the software by the group facilitators.

A lively discussion developed only in two of the three groups. Here, participants started to discuss what settings to modify and assessed the outcomes, e.g. the influence of persons in a tramway on the emissions per person-kilometer. In the third group, adverse group dynamic processes led most participants to refuse working with the model at all.

No major problems were reported with the GUI or the result visualization, although most participants let the facilitators operate the software instead of doing it themselves. The main reason for this was a general time constraint, which did not leave enough time to learn using the GUI for users unfamiliar with computers. However, those users who ran the model themselves were most enthusiastic about the model and its opportunities.

The results caused diverse reactions. For many users, the observed difference of emissions between tramways and mopeds was so surprising that they questioned the credibility of the model, although a straightforward explanation (increased energy use due to vehicle weight) had been provided. Most of the results, however, appeared to be trivial or well known (cars emit relatively more than buses). This raised general questions on the added value from using the model. Model-specific shortcomings (missing suburban train) also led to a disqualification of the model by some users.

Interestingly, the model did rarely cause participants to reflect on their own mobility behavior. This is probably due to an improper timing within the INNOCOPE workshop series, which put EcoClass into a phase that dealt with design issues in detail. Here, a full-featured LCA tool would have been needed, while EcoClass might have been more appropriate to appear in the first workshop to raise awareness on mobility issues.

\section{Conclusions}

The EcoClass model provides environmental impact data for participatory product development based on the GEMIS life cycle assessment database. EcoClass introduces utilization processes as a new process category. These allow for easy online variation of the product utilization phase even by inexperienced users, in contrast to standard LCA tools where different utilization schemes require the definition of different processes. Since this increased flexibility comes at the cost of slightly reduced accuracy, EcoClass is intended to give an introductory overview on environmental impacts from product utilization. Currently, EcoClass is restricted to urban person- 
nel transport systems and still lacks much of the functionality of fully featured LCA tools; these restrictions shall be dropped in future versions.

The first use of EcoClass during a pilot study showed that much of the design goals have been reached: Most users approached the GUI with minor efforts and understood the graphical results intuitively. The results stimulated discussions among the participants that focused on data credibility and the usefulness of models in general. However, participants were rarely observed to reflect on their personal mobility behavior.

Future uses of EcoClass in its current version should concentrate on raising awareness. This might include introductory sessions in workshop series, but could also be extended to demonstrations at fairs, public information desks, or in schools. Additional fields of use will open up for future model versions that offer full LCA functionality.

\section{Acknowledgements}

We thank our colleagues in the GELENA project for their feedback and suggestions during the development of EcoClass. Generous funding of the GELENA project by the German Federal Ministry for Education and Research (BMBF) is gratefully acknowledged.

\section{References}

Fritsche U, Rausch L, Simon K-H (1989): Umweltwirkungsanalyse von Energiesystemen: Gesamt-Emissions-Modell Integrierter Systeme (GEMIS), Endbericht, Öko-Institut Darmstadt /WZ III Gesamthochschule Kassel

Fritsche UR, Leuchtner J, Matthes FC, Rausch L, Simon K-H (1994): GesamtEmissions-Modell Integrierter Systeme (GEMIS) Version 2.1: Aktualisierter und erweiterter Endbericht, Darmstadt/Freiburg/Berlin/Kassel

Hansen U, Raabe T (1991): Konsumentenbeteiligung an der Produktentwicklung von Konsumgütern. Ergebnisse einer empirischen Untersuchung, Zeitschrift für Betriebswirtschaft 61(2), 171-194

Hoffmann E (2006): Consumer-Integration in Sustainable Product Development, paper presented at the 13th International Conference of The Greening of Industry Network, 02.-05.07.2006, Cardiff, UK

Kasemir B, Jäger J, Jaeger CC, Gardner MT (eds.) (2003): Public Participation in Sustainability Science, Cambridge Univ. Press, Cambridge MA

Lester RK, Piore MJ, Malek KM (1998): Interpretative Management: What General Managers Can Learn From Design, Harvard Business Review (MarchApril 1998), 86-96

van den Ven A (1986): Central Problems in the Management of Innovation, Management Science 32(5), 569-607 